\title{
Genetic polymorphisms in the TERT-CLPTM1L region and lung cancer susceptibility in Chinese males
}

\author{
XUYANG XIAO $^{1}$ and WUBIN $\mathrm{HE}^{2}$ \\ ${ }^{1}$ Department of Thoracic Surgery and ${ }^{2}$ Biological Therapy Center Laboratory, The First Affiliated Hospital \\ of Jinzhou Medical University, Jinzhou, Liaoning 121001, P.R. China
}

Received January 25, 2017; Accepted May 18, 2017

DOI: $10.3892 / \mathrm{ol} .2017 .6289$

\begin{abstract}
The objective of the present study was to analyze the relationship between genetic polymorphisms of the rs 2736098 locus of the telomerase reverse transcriptase (TERT) gene and the rs401681 locus of the cleft lip and palate transmembrane protein 1 (CLPTM1L) gene and the risk of developing lung cancer in males in Jinzhou. A total of 214 lung cancer patients who were admitted in Jinzhou Medical University were analyzed, and 216 healthy males were selected as controls. Venous blood from all subjects and data on relevant risk factors were collected. DNA was extracted from peripheral blood by the phenol-chloroform method. Real-time fluorescent quantitative PCR (TaqMan real-time PCR) was used for DNA amplification. The genotyping results of the genetic polymorphisms of the TERT rs2736098 and CLPTM1L rs401681 loci were detected. The risk of developing lung cancer in the population with the TERT rs2736098 locus carrying the T allele was 1.614 times that with the TERT rs2736098 locus carrying the Callele after adjustment of the age factor. The risk of developing lung cancer in the population carrying the TT mutant genotype and the CT genotype increased significantly compared with that carrying the $\mathrm{CC}$ wild genotype [odds ratio $(\mathrm{OR})=1.815$, 95\% CI=1.132-2.957; OR=2.417, 95\% CI=1.158-4.943]. Based on a comparison between the combination of the two mutant genotypes (CT+TT) and the wild homozygous genotype (CC), the mutant genotype increased the risk of developing lung cancer $(\mathrm{OR}=1.955,95 \% \mathrm{CI}=1.213-3.157)$. The risk of developing lung cancer in the population with the CLPTM1L rs401681 locus carrying the $\mathrm{T}$ allele was 1.399 times that carrying the $\mathrm{C}$ allele $(\mathrm{OR}=1.343,95 \% \mathrm{CI}=1.035-1.978)$. The population with the TERT rs 2736098 locus carrying the mutant genotype $(\mathrm{CT}+\mathrm{TT})$ was associated with the number of tumors
\end{abstract}

Correspondence to: Dr Wubin He, Biological Therapy Center Laboratory, The First Affiliated Hospital of Jinzhou Medical University, 2 People's Street, Guta, Jinzhou, Liaoning 121001, P.R. China

E-mail:w0f8v5@163.com

Key words: telomerase reverse transcriptase, cleft lip and palate transmembrane protein 1 , lung cancer, mononucleotide polymorphism, hereditary susceptibility
$(\mathrm{OR}=0.553,95 \% \mathrm{CI}=0.236-0.928)$. In conclusion, in males, the TERT rs2736098 and CLPTM1L rs401681 T alleles are the susceptibility factors for developing lung cancer. Individuals, including the smoking population, who carry both the TERT rs2736098 and CLPTM1L rs401681 T alleles are more likely to develop lung cancer.

\section{Introduction}

Tumorigenesis is a multi-factor, multi-stage, and multi-gene process. In addition to environmental factors, dietary factors, biological factors, and hereditary factors play important roles in tumor progression $(1,2)$. Genetic susceptibility is determined by a single nucleotide on the individual genome $(3,4)$. During tumorigenesis, environmental factors play an initiating role, while hereditary factors determine the susceptibility of individuals to tumorigenesis. In recent years, research on genetic susceptibility to tumors has attracted much attention. Recent research in molecular epidemiology has aimed to identify and determine the mutant loci of tumor-related genes, and further study the interactions between the environment and genes, and the interactions between genes by studying mononucleotide polymorphisms (4).

Within a DNA sequence, polymorphisms arising from mutation of a single nucleotide (A, T, C, and G) are called single nucleotide polymorphisms (SNPs) (5). SNPs are a major form of genome polymorphism (6). The gene coding region of an SNP is called a coding region SNP (cSNP), which may lead to changes in the amino acid sequence of proteins, and changes in protein function. cSNPs have important biological significance (7). Based on estimation, 1 in 1,000 people have one SNP in the genome. The 3 billion human basic groups have a total of over 3 million SNPs (8). Different individuals have different susceptibilities to carcinogenic factors in the environment because of polymorphisms. Therefore, SNPs can be used to screen populations susceptible to tumors in a rapid and large-scale manner. Research on SNPs related to tumors has also been a focus in molecular epidemiology in recent years. The chromosome 5 p15.33 region has two known genes, human telomerase reverse transcriptase (TERT) and cleft lip and palate transmembrane protein 1 (CLPTM1L) (9-11). These genes have multiple cancer risk loci and may be associated with tumorigenesis.

TERT is the catalytic subunit of telomerase, and is located on chromosome $5 \mathrm{p} 15.33$. It spans $35 \mathrm{~kb}$, and comprises 16 exons 
and 15 introns. It is a rate-limiting factor for synthesizing the telomerase holoenzyme (12). Its expression correlates with the expression of telomerase. Changes in TERT can also reflect corresponding changes in the activity of intracellular telomerase (13). TERT is an important target site for inhibiting telomerase activity. TERT can increase the risk of tumors by influencing telomerase activity and telomere length. In addition, telomerase participates in processes such as apoptosis, DNA damage repair, and regulation of gene expression, thus influencing tumorigenesis and progression $(14,15)$.

The CLPTM1L gene encodes for a transmembrane protein, which induces apoptosis in cisplatin-resistant cell lines. It is expressed in multiple normal and malignant tissues such as the skin, lung, ovary, cervix, and thymus $(16,17)$. However, the functions of CLPTM1L, and its role in tumorigenesis remain unclear. At present, CLPTM1L has been confirmed to be overexpressed in human ovarian tumor cell lines and is resistant to cisplatin. CLPTM1L overexpression in pulmonary tumor cells can protect them from apoptosis induced by genotoxic stress, indicating that it may have antiapoptotic function. CLPTM1L is also highly expressed in other tumor tissues. The aforementioned observations suggest that CLPTM1L may be associated with the genesis of multiple tumor types. The genome-wide association study reported that the SNPs, TERT rs2736098 and CLPTM1L rs401681, are significantly correlated with cancer risk (18).

Regarding research on the relationship between genetic polymorphisms of specific loci and lung cancer susceptibility, Zhang et al studied the relationship between the genetic polymorphism at rs2736098, and the risk of developing lung cancer in 400 lung cancer patients and 400 healthy subjects (controls) in the Asian population in 2012. Their results showed that genetic mutation at the rs2736098 locus increases the risk of developing lung cancer (19). Currently, there is no research on the relationship between the rs401681 locus and the risk of developing lung cancer.

Using a case-control design method, the aim of the present study was to analyze the relationship between the genetic polymorphism of the rs2736098 locus of the TERT gene and the rs401681 locus of the CLPTM1L gene and the risk of developing lung cancer in males in Jinzhou city.

\section{Patients and methods}

Selection of research subjects. Male patients definitely diagnosed with lung cancer based on histopathology who received operative treatment in Grade 3, Class A hospitals in Jinzhou city were selected, with a case-control research method, and assigned to the case group. Inclusion criteria for the case group: i) male; ii) primary lung cancer, excluding metastatic lung cancer and iii) received no chemotherapy or radioactive therapy. A total of 201 lung cancer patients aged from 30 to 87 years who sought medical advice in Grade 3, Class A hospitals in Jinzhou city from 2008 to 2013 were included as research subjects. In addition, 211 healthy males who underwent physical examinations in the physical examination center during the corresponding period were selected as controls using the frequency matching method. All research subjects signed an informed consent form. Next, blood samples from all research subjects were collected. Data on demographic characteristics, relevant clinical indexes, and exposure to environmental factors were obtained by questionnaires. This study was approved by the Ethics Committee of the First Affiliated Hospital of Jinzhou Medical University. Signed written informed consents were obtained from all participants before the study.

Epidemiological investigation. Uniformly prepared questionnaires were used. An epidemiological investigation into the research subjects was made by two trained investigators. The content of the investigation included demographic data (names and ages), smoking history, and clinical indexes. Non-smokers referred to individuals who smoked $<100$ cigarettes in their lives. The relevant clinical indexes were grouped according to the range of standard reference values. All investigation data were checked repeatedly and coded. A database was established with the EpiData software version 3.5 (Tree star Inc., Ashland, OR, USA), and data were entered.

DNA extraction. Frozen anticoagulated blood from lung cancer patients was thawed in a water bath at $37^{\circ} \mathrm{C}$. A total of $1 \mathrm{ml}$ of whole blood was taken, and $4 \mathrm{ml}$ of erythrocyte lysis buffer (Sigma-Aldrich, St. Louis, MO, USA) was added. Samples were shaken sufficiently, mixed well, and allowed to stand for $30 \mathrm{~min}$. Samples were centrifuged at $698.75 \mathrm{x} \mathrm{g}$, and the supernatant was discarded. A total of $1 \mathrm{ml}$ of the buffer + $11 \mathrm{ml}$ protease $\mathrm{K}$ (Qiagen, Valencia, CA, USA) were added and shaken in a water bath for $20 \mathrm{~h}$ at $37^{\circ} \mathrm{C}$ until the leukocyte blocks disappeared. An equal volume of balance phenol (Qiagen) was added. Samples were inverted gently for $10 \mathrm{~min}$, and centrifuged at $1,006.2 \mathrm{x} \mathrm{g}$. The supernatant was pipetted into $5 \mathrm{ml}$ EP tubes. This step was repeated once. An equal amount of 24:1 chloroform-isoamylol (Promega Corporation, Madison, WI, USA) was added. Samples were shaken gently for $10 \mathrm{~min}$, and centrifuged at $1,006.2 \mathrm{x} \mathrm{g}$. The supernatant was then pipetted. This step was repeated once. The supernatant was pipetted into $2 \mathrm{ml}$ EP tubes. Cold anhydrous ethanol was added (Beijing Chemical Reagent Co., Ltd., Beijing, China). Samples were centrifuged at $10,500 \mathrm{x} \mathrm{g}$ for $10 \mathrm{~min}$. The supernatant was discarded. A total of $200 \mathrm{ml}$ of $70 \%$ ethanol was added. Samples were washed twice. Samples were then inverted slowly for $1 \mathrm{~min}$, and centrifuged at $10,500 \mathrm{x} \mathrm{g}$ for $10 \mathrm{~min}$. The supernatant was discarded. Samples were air dried at room temperature. A total of $100 \mathrm{ml}$ of TE was added to dissolve the DNA. The OD value (Thermo Fisher Scientific, Waltham, MA, USA) was measured. Samples were placed at $-20^{\circ} \mathrm{C}$.

Real-time PCR genotyping. The TaqMan genotyping technique was used for the detection of TERT rs2736098 and CLPTM1L rs401681 polymorphisms. The PCR TaqMan probe and primers were designed and synthesized by ABI PE Applied Biosystems (Foster City, CA, USA). TaqMan Master mix was from ABI PE Applied Biosystems. PCR reaction system: $2.50 \mu \mathrm{l}$ of $2 \mathrm{x}$ TaqMan Master mix, $0.25 \mu \mathrm{l}$ of 20x primer and probe mixture, $1.25 \mu \mathrm{l}$ of $\mathrm{H}_{2} \mathrm{O}$, and $1.00 \mu \mathrm{l}$ of DNA, for a total of $5.00 \mu \mathrm{l}$. PCR amplification conditions: PCR reactions and fluorescence signal reading were performed on an ABI 7500 fluorescent quantitation PCR amplifier (ABI PE Applied Biosystems). The amplification conditions were as 
Table I. Basic information on the case group and control group.

\begin{tabular}{lcc}
\hline Characteristics & Case group $(\%)$ & Control group (\%) \\
\hline Males & 214 & 216 \\
Average age & $56.36 \pm 10.43$ & $57.27 \pm 12.32$ \\
Smokers & $130(60.7)$ & $82(34.1)$ \\
Non-smokers & $84(39.3)$ & \\
CRP & $161(75.23)$ & \\
SCCA (mg/l) & $56(26.1)$ & \\
CEA (mg/l) & $78(36.4)$ & \\
TNM stage I+II & $90(42.0)$ & \\
TNM stage III+IV & $124(58.0)$ & \\
No lymphatic metastasis & $182(85.0)$ & \\
Lymphatic metastasis & $32(15.0)$ & \\
No. of tumors & $105(49.0)$ & \\
$=1$ & $99(51.0)$ & \\
$\geq 2$ & & \\
\hline
\end{tabular}

${ }^{a}$ Two-sided test; the differences were considered statistically significant when $\mathrm{P}<0.05$. CRP, C-reactive protein; SCCA, squamous cell carcinoma antigen; CEA, carcinoembryonic antigen; TNM, tumor-node-metastasis.

follows: pre-denaturation for $10 \mathrm{~min}$ at $95^{\circ} \mathrm{C}$; denaturation for $30 \mathrm{sec}$ at $92^{\circ} \mathrm{C}$, annealing and extension for $1 \mathrm{~min}$ at $60^{\circ} \mathrm{C}$, for 47 cycles in total. SDS software version 4.0 (ABI PE Applied Biosystems) was used for genotype analysis. The genotypes of samples were judged by detecting FAM and VIC fluorescence intensity marked by different alleles of the same gene.

Statistical analysis. SPSS 19.0 software (SPSS, Inc., Chicago, IL, USA) was used for data analysis. A two-sided test was conducted to detect the differences in distribution of demographic characteristics, relevant risk factors, and SNP genotype between the case group and control group. $\mathrm{P}<0.05$ was considered statistically significant. The logistic regression model was used to compute the odds ratio (OR) of various factors and their $95 \%$ confidence interval $(95 \% \mathrm{CI})$, and analyze the relationship between genetic polymorphisms and the risk of developing lung cancer.

\section{Results}

General conditions of research subjects. The basic conditions of the case group and control group are shown in Table I. A total of 214 male lung cancer patients and 216 healthy males were included as research subjects. The ages of the case group and control group ranged from 30 to 86 years. The average age of the case group was $56.36 \pm 10.43$ years, and the average age of the control group was $57.27 \pm 12.32$ years. There were no significant differences in age between the two groups $(\mathrm{P}=0.513)$. Based on a statistical analysis, there were no significant differences in distribution of patients with or without a history of smoking between the case group and control group $(\mathrm{P}=0.256)$. The distribution of relevant clinical indexes of the lung cancer patients [carcinoembryonic antigen, tumor-nodemetastasis (TNM) staging, lymphatic metastasis, and number of tumors] is shown in Table I.

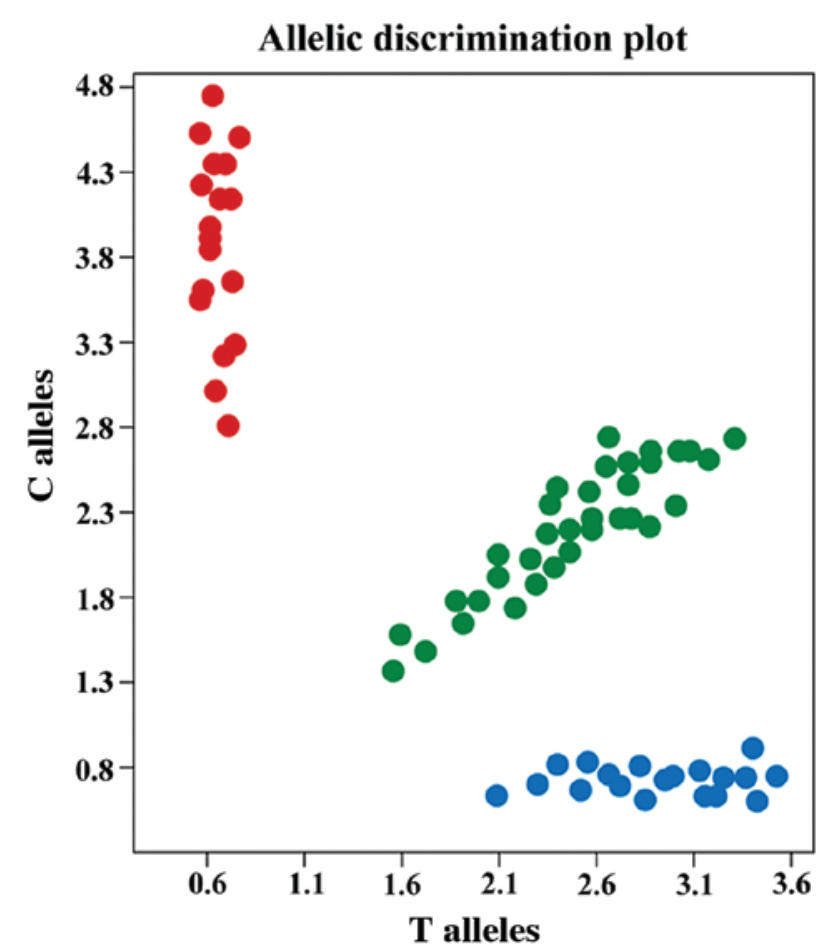

Figure 1. Detection of the genotype of the TERT rs2736098 locus. The red points represent $\mathrm{CC}$ homozygous individuals. The blue points represent TT homozygous individuals. The green points represent CT heterozygous individuals. TERT, telomerase reverse transcriptase.

Distribution of TERT and CLPTMIL polymorphism genotypes and the relationship between the distribution and risk of developing lung cancer in males. The genotypes of the TERT rs2736098 and CLPTM1L rs401681 loci are shown in Fig. 1. The vertical coordinates represent the $\mathrm{C}$ alleles, and the horizontal coordinates represent the $\mathrm{T}$ alleles. 
Table II. Relationship between the genetic polymorphism of TERT and CLPM1L and lung cancer susceptibility in males.

\begin{tabular}{|c|c|c|c|c|}
\hline Genotype & Case group & Control group & $\mathrm{P}$-value & OR $(95 \% \mathrm{CI})^{\mathrm{a}}$ \\
\hline \multicolumn{5}{|c|}{ TERT rs 2736098} \\
\hline $\mathrm{CC}$ & 78 & 123 & & 1.0 \\
\hline $\mathrm{CT}$ & 95 & 77 & 0.013 & $1.815(1.132-2.957)$ \\
\hline $\mathrm{TT}$ & 30 & 25 & 0.014 & $2.417(1.158-4.943)$ \\
\hline $\mathrm{CT}+\mathrm{TT}$ & 129 & 101 & 0.003 & $1.955(1.213-3.157)$ \\
\hline $\mathrm{T}$ allele & & & 0.005 & $1.614(1.134-2.267)$ \\
\hline \multicolumn{5}{|c|}{ CLPTM1L rs401681 } \\
\hline $\mathrm{CC}$ & 85 & 128 & & 1.0 \\
\hline $\mathrm{CT}$ & 99 & 79 & 0.015 & $1.732(1.121-2.857)$ \\
\hline $\mathrm{TT}$ & 15 & 13 & 0.338 & $1.532(0.651-3.546)$ \\
\hline $\mathrm{CT}+\mathrm{TT}$ & 112 & 92 & 0.019 & $1.744(1.151-2.736)$ \\
\hline $\mathrm{T}$ allele & & & 0.044 & $1.343(1.035-1.978)$ \\
\hline
\end{tabular}

${ }^{a}$ The OR value was subjected to age correction. The differences were considered statistically significant at $\mathrm{P}<0.05$. TERT, telomerase reverse transcriptase; CLPTM1L, cleft lip and palate transmembrane protein 1; OR, odds ratio.

Table III. Relationship between the joint action of the genetic polymorphism of TERT and CLPTM1L and lung cancer susceptibility in males.

\begin{tabular}{lccccc}
\hline TERT rs2736098 & CLPTM1L rs401681 & Case group & Control group & P-value & OR (95\% CI ${ }^{\mathrm{a}}$ \\
\hline $\mathrm{CC}$ & $\mathrm{CC}$ & 23 & 6 & & \\
$\mathrm{CC}$ & $\mathrm{CT}+\mathrm{TT}$ & 52 & 67 & 0.058 & $1.752(0.944-3.267)$ \\
$\mathrm{CT}+\mathrm{TT}$ & $\mathrm{CC}$ & 66 & 66 & 0.043 & $1.823(1.177-3.236)$ \\
$\mathrm{CT}+\mathrm{TT}$ & $\mathrm{CT}+\mathrm{TT}$ & 63 & 30 & $<0.001$ & $4.457(2.337-8.358)$ \\
\hline
\end{tabular}

${ }^{a}$ The OR value was subjected to age correction. The differences were considered statistically significant at $\mathrm{P}<0.05$. TERT, telomerase reverse transcriptase; CLPTM1L, cleft lip and palate transmembrane protein 1; OR, odds ratio.

Relationship between the genetic polymorphisms of TERT and CLPTMIL and lung cancer susceptibility in males. The genotype frequencies of the two genetic loci, TERT rs2736098 and CLPTM1L rs401681, in both the case group and control group complied with Hardy-Weinberg equilibrium. The polymorphisms of the two genetic loci and the distribution of the alleles in both groups are shown in Table II. The results showed that the risk of developing lung cancer in the population with the TERT rs2736098 locus carrying the $\mathrm{T}$ allele was 1.614 times the risk of developing lung cancer in the population carrying the $\mathrm{C}$ allele $(\mathrm{OR}=1.614,95 \% \mathrm{CI}=1.134-2.267)$ following adjustment of the age factor. Furthermore, the risk of developing lung cancer in the population carrying the TT mutant gene and the CT genotype increased significantly compared with that in the population carrying the $\mathrm{CC}$ wild genotype. The differences were statistically significant $(\mathrm{OR}=1.815$, 95\% CI=1.132-2.957); $(\mathrm{OR}=2.417,95 \% \mathrm{CI}=1.158-4.943)$. In addition, based on a comparison between the combination of two mutant genotypes (CT+TT) and the wild homozygous genotype (CC), the mutant genotype increased the risk of developing lung cancer. The differences were statistically significant $(\mathrm{OR}=1.955,95 \% \mathrm{CI}=1.213-3.157)$. The risk of developing lung cancer in the population carrying the $\mathrm{T}$ allele at the CLPTM1L rs401681 locus was 1.399 times the risk of developing lung cancer in the population carrying the $\mathrm{C}$ allele $(\mathrm{OR}=1.343$, 95\% $\mathrm{CI}=1.035-1.978)$. Based on a comparison between the combination of two mutant genotypes (CT+TT) and the wild homozygous (CC) genotype, the mutant genotype increased the risk of developing lung cancer. The differences were statistically significant $(\mathrm{OR}=1.744,95 \% \mathrm{CI}=1.151-2.736)$.

Next, the genetic polymorphisms of the TERT rs2736098 and CLPTM1L rs401681 loci were combined to analyze the joint action of the two genes. The results of analysis are shown in Table III. The risk of developing lung cancer in individuals with mutant genotypes of the TERT rs2736098 and CLPTM1L rs401681 loci increased significantly (OR=4.457, 95\% CI=2.337-8.358). The differences were statistically significant.

The results in Table IV show that individuals carrying the mutant genotypes (CT+TT), CC of TERT rs2736098, and mutant genotype (CT+TT) of CLPTMIL rs401681 in the smoking population had a significantly higher risk of developing lung cancer compared with those carrying the wild homozygous genotype (CC) in the non-smoking population $(\mathrm{OR}=2.348$, 95\% CI=1.156-4.347; OR=2.785, 95\% CI=1.254-6.057). The differences were statistically significant. 
Table IV. Relationship between the joint action of genetic polymorphism of TERT and CLPTM1L and smoking, and lung cancer susceptibility in males.

\begin{tabular}{|c|c|c|c|c|c|}
\hline Smokers & Genotype & Case group & Control group & P-value & OR $(95 \% \mathrm{CI})^{\mathrm{a}}$ \\
\hline \multicolumn{6}{|c|}{ TERT rs2736098 } \\
\hline- & $\mathrm{CC}$ & 38 & 65 & & 1.0 \\
\hline- & $\mathrm{CT}+\mathrm{TT}$ & 47 & 72 & 0.543 & $1.255(0.658-2.257)$ \\
\hline+ & $\mathrm{CC}$ & 25 & 41 & 0.755 & $0.815(0.422-1.868)$ \\
\hline+ & $\mathrm{CT}+\mathrm{TT}$ & 33 & 26 & 0.028 & $2.348(1.156-4.347)$ \\
\hline \multicolumn{6}{|c|}{ CLPTM1L rs401681 } \\
\hline- & $\mathrm{CC}$ & 36 & 73 & & 1.0 \\
\hline- & $\mathrm{CT}+\mathrm{TT}$ & 45 & 61 & 0.159 & $1.533(0.819-2.917)$ \\
\hline+ & $\mathrm{CC}$ & 25 & 43 & 0.816 & $1.048(0.532-2.147)$ \\
\hline+ & $\mathrm{CT}+\mathrm{TT}$ & 32 & 28 & 0.008 & $2.785(1.254-6.057)$ \\
\hline
\end{tabular}

${ }^{a}$ The OR value was subjected to age correction. The differences were considered statistically significant at $\mathrm{P}<0.05$. TERT, telomerase reverse transcriptase; CLPTM1L, cleft lip and palate transmembrane protein 1; OR, odds ratio.

Table V. Analysis of the correlation between the genetic polymorphism of the TERT rs2736098 locus and the progression of course of disease of the male lung cancer patients.

\begin{tabular}{lcccc}
\hline & \multicolumn{2}{c}{ Indexes of progression of course of disease } & & \\
\cline { 2 - 4 } Genotype & I+II & III+IV & P-value & OR $(95 \% \text { CI })^{\mathrm{a}}$ \\
\hline CC & 31 & 44 & 1.0 & \\
CT & 53 & 45 & 0.547 & $0.144(0.351-1.163)$ \\
TT & 12 & 18 & 0.768 & $0.644(0.334-1.945)$ \\
CT+TT & 61 & 65 & 0.148 & $0.655(0.348-1.219)$ \\
\hline & Lymphatic metastasis $(-)$ & Lymphatic metastasis $(+)$ & & \\
\hline CC & 67 & 13 & 1.0 & \\
CT & 89 & 10 & 0.558 & $0.256(0.243-1.533)$ \\
TT & 28 & 15 & 0.947 & $0.919(0.255-3.153)$ \\
CT+TT & 115 & 0.358 & $0.655(0.259-1.519)$ \\
\hline & More than two tumors & & \\
\hline CC & One tumor & 44 & 1.0 & \\
CT & 30 & 44 & 0.543 & $0.047(0.292-0.991)$ \\
TT & 56 & 12 & 0.435 & $0.058(0.193-1.054)$ \\
CT+TT & 17 & 55 & 0.021 & $0.553(0.236-0.928)$ \\
\hline
\end{tabular}

${ }^{a}$ The OR value was subjected to age correction. The differences were considered statistically significant at $\mathrm{P}<0.05$. TERT, telomerase reverse transcriptase; OR, odds ratio.

Analysis of the correlation between the genetic polymorphism of TERT and CLPTMIL and progression of lung cancer. The results in Tables V and VI show that in lung cancer patients, carrying the mutant genotype $(\mathrm{CT}+\mathrm{TT})$ at the TERT rs2736098 locus is associated with the number of tumors $(\mathrm{OR}=0.553,95 \% \mathrm{CI}=0.236-0.928)$. The differences were statistically significant. The indexes related to progression of other lung cancers (TNM staging and lymphatic metastasis) were not correlated with the genetic polymorphism.

\section{Discussion}

The incidence of lung cancer is a multi-factor and multi-stage process caused by genetic and environmental factors (1). In recent years, numerous studies have focused on the 
Table VI. Analysis of the correlation between the genetic polymorphism of the CLPTM1L rs401681 locus and the progression of the course of disease of male lung cancer patients.

Indexes of progression of course of disease

\begin{tabular}{lrccc}
\cline { 2 - 3 } Genotype & I+II & III+IV & P-value & OR $(95 \% \text { CI })^{\mathrm{a}}$ \\
\hline CC & 37 & 45 & 1.0 & \\
CT & 44 & 47 & 0.775 & $0.445(0.457-1.423)$ \\
TT & 5 & 13 & 1.432 & $0.512(0.443-4.257)$ \\
CT+TT & 51 & 66 & 0.618 & $0.855(0.432-1.523)$ \\
\hline
\end{tabular}

\begin{tabular}{lcccc}
\hline & Lymphatic metastasis (-) & Lymphatic metastasis $(+)$ & & \\
\hline CC & 76 & 10 & 1.0 & \\
CT & 80 & 13 & 0.858 & $1.076(0.438-2.756)$ \\
TT & 15 & 6 & 0.144 & $2.689(0.718-9.832)$ \\
CT+TT & 115 & 11 & 0.545 & $1.227(0.554-3.038)$ \\
\hline
\end{tabular}

\begin{tabular}{lcccc}
\hline & One tumor & More than two tumors & & \\
\hline CC & 45 & 36 & 1.0 & \\
CT & 43 & 55 & 1.458 & $0.224(0.853-2.534)$ \\
TT & 6 & 10 & 1.459 & $0.434(0.538-4.267)$ \\
CT+TT & 56 & 65 & 0.216 & $1.455(0.832-2.566)$ \\
\hline
\end{tabular}

${ }^{a}$ The OR value was subjected to age correction. The differences were considered statistically significant at P<0.05. CLPTM1L, cleft lip and palate transmembrane protein 1 ; OR, odds ratio.

relationship between relevant genetic polymorphisms and lung cancer susceptibility. Studying males from Jinzhou city as research subjects, we investigated the relationship between the risk of developing lung cancer and polymorphisms of TERT and CLPTM1L as well as exposure to risk factors. Both SNPs at the TERT rs2736098 locus and CLPTM1L rs401681 locus can increase the risk of developing lung cancer. The interactions between the two factors and genetic and environmental factors significantly increase the risk of developing lung cancer.

TERT is the catalytic subunit of telomerase, and is located on chromosome $5 \mathrm{p} 15.33$. It spans $35 \mathrm{~kb}$, and comprises 16 exons and 15 introns. It is a rate-limiting factor for synthesizing the telomerase holoenzyme. The rs 2736098 locus is located on the second exon of the gene. The molecular weight of the TERT protein is approximately $127 \mathrm{kDa}$, and is primarily distributed in the nucleus. Mutation of its non-active structural domain, DAT, influences the binding between TERT and telomere DNA, thus affecting the activity of telomerase in extending telomeres. Moreover, it has higher tumor specificity (19). Our results showed that there were significant differences in genotype frequencies between the case group and control group. The risk of developing lung cancer in the population carrying the $\mathrm{T}$ allele is increased significantly compared with the population carrying the $\mathrm{C}$ allele. The risk of developing lung cancer in the population carrying the mutant genotype and the CT genotype is increased significantly compared with the population carrying the $\mathrm{CC}$ wild genotype. The differences were statistically significant. These results indicate that genetic polymorphism of the TERT rs2736098 locus may be an independent risk factor for the incidence of lung cancer in males. CLPTM1L is located on the chromosome 5p15.33 region, and encodes for a transmembrane protein. It can induce cell apoptosis in cisplatin-resistant cell lines. It is expressed in multiple normal and malignant tissues such as the skin, lung, ovary, cervix, and thymus (20,21). Currently, CLPTM1L is confirmed to be overexpressed and resistant to cisplatin in human ovarian tumor cell lines. Its overexpression in pulmonary tumor cells can protect against apoptosis induced by genotoxic stress (22-24), indicating that it has anti-apoptotic function. However, the functions of CLPTM1L, and its role in tumorigenesis remain unclear. We studied the relationship between the genetic polymorphism of the CLPTM1L rs401681 locus and lung cancer in males. The risk of developing lung cancer in the population carrying the $\mathrm{T}$ allele is increased significantly compared with those carrying the $\mathrm{C}$ allele. Based on a comparison between the combination of the two mutant genotypes (CT+TT) and the wild homozygous genotype (CC), the mutant genotype increases the risk of developing lung cancer. There is no research on the relationship between the genetic polymorphism and lung cancer, whereas a great deal on lung cancer, but there are differences among the results.

This study had limitations. The sample-size was relatively small. The analysis only involved SNPs of two genes in the chromosome 5p15.33 region. Future studies require an expanded population range and increased sample-size. Furthermore, the analysis should involve multiple genetic loci. The relationship between genetic polymorphisms of the 
rs2736098 and rs401681 loci and the progression of the disease requires further validation in future studies.

In conclusion, there is a correlation between genetic polymorphisms of TERT and CLPTM1L and lung cancer in males. The TERT rs2736098 and CLPTM1L rs401681T alleles increase the risk of developing lung cancer in males. They play a role in understanding the pathogenesis of lung cancer and screening for the high-risk population.

\section{Acknowledgements}

The present study was funded by Jinzhou Medical University special fund XZJJ20140216.

\section{References}

1. Yin J, Li Y, Yin M, Sun J, Liu L, Qin Q, Li X, Long L, Nie S and Wei S: TERT-CLPTM1L polymorphism rs401681 contributes to cancers risk: evidence from a meta-analysis based on 29 publications. PLoS One 7: e50650, 2012.

2. Mocellin S, Verdi D, Pooley KA, Landi MT, Egan KM, Baird DM, Prescott J, De Vivo I and Nitti D: Telomerase reverse transcriptase locus polymorphisms and cancer risk: a field synopsis and meta-analysis. J Natl Cancer Inst 104: 840-854, 2012.

3. Chen XF, Cai S, Chen QG, Ni ZH, Tang JH, Xu DW and Wang XB: Multiple variants of TERT and CLPTM1L constitute risk factors for lung adenocarcinoma. Genet Mol Res 11: 370-378, 2012.

4. Jiang M, Wu H and Qin C: Genetic variant rs401681 at 5p15.33 modifies susceptibility to lung cancer but not esophageal squamous cell carcinoma. PLoS One 8: e84277, 2013.

5. Yang IA, Holloway JW and Fong KM: Genetic susceptibility to lung cancer and co-morbidities. J Thorac Dis 5 (Suppl 5): 454-462, 2013

6. Zhong R, Liu L, Zou L, Zhu Y, Chen W, Zhu B, Shen N, Rui R, Long L, Ke J, et al: Genetic variations in TERT-CLPTM1L locus are associated with risk of lung cancer in Chinese population. Mol Carcinog 52 (Suppl 1): E118-E126, 2013.

7. Li C, Yin Z, Wu W, Li X, Ren Y and Zhou B: Genetic variations in TERT-CLPTM1L genes and risk of lung cancer in Chinese women nonsmokers. PLoS One 8: e64988, 2013.

8. Lan Q, Cawthon R, Gao Y,Hu W, Hosgood HD III, Barone-Adesi F, Ji BT, Bassig B, Chow WH, Shu X, et al: Longer telomere length in peripheral white blood cells is associated with risk of lung cancer and the rs2736100 (CLPTM1L-TERT) polymorphism in a prospective cohort study among women in China. PLoS One 8: e59230, 2013.

9. Myneni AA, Chang SC, Niu R, Liu L, Ochs-Balcom HM, Li Y, Zhang C, Zhao B, Shi J, Han X, et al: Genetic polymorphisms of TERT and CLPTM1L and risk of lung cancer - a case-control study in a Chinese population. Lung Cancer 80: 131-137, 2013.

10. Zhao Z, Li C, Yang L, Zhang X, Zhao X, Song X, Li X, Wang J, Qian J, Yang Y, et al: Significant association of 5p15.33 (TERT-CLPTM1L genes) with lung cancer in Chinese Han population. Exp Lung Res 39: 91-98, 2013.

11. Wang H, Zhao Y, Ma J, Zhang G, Mu Y, Qi G, Fang Z, Wang L, Fan Q and Ma Z: The genetic variant rs401681C/T is associated with the risk of non-small cell lung cancer in a Chinese mainland population. Genet Mol Res 12: 67-73, 2013.
12. Tseng TS, Park JY, Zabaleta J, Moody-Thomas S, Sothern MS, Chen T, Evans DE and Lin HY: Role of nicotine dependence on the relationship between variants in the nicotinic receptor genes and risk of lung adenocarcinoma. PLoS One 9: e107268, 2014.

13. Yin Z, Cui Z, Ren Y, Zhang H, Yan Y, Zhao Y, Ma R, Wang Q, He Q and Zhou B: Genetic polymorphisms of TERT and CLPTM1L, cooking oil fume exposure, and risk of lung cancer: a case-control study in a Chinese non-smoking female population. Med Oncol 31: 114, 2014.

14. Wang Z,Zhu B, Zhang M,Parikh H, Jia J, Chung CC, Sampson JN, Hoskins JW, Hutchinson A, Burdette L, et al: Imputation and subset-based association analysis across different cancer types identifies multiple independent risk loci in the TERT-CLPTM1L region on chromosome 5p15.33. Hum Mol Genet 23: 6616-6633, 2014.

15. Zhang Y, Zhao M, Shen L, Ren Y, Su L, Li X, Yin Z and Zhou B: Genetic polymorphisms of TERT and CLPTM1L and risk of lung cancer: a case-control study in northeast Chinese male population. Med Oncol 31: 18, 2014.

16. Zhao MM, Zhang Y, Shen L, Ren YW, Li XL, Yin ZH and Zhou BS: Genetic variations in TERT-CLPTM1L genes and risk of lung cancer in a Chinese population. Asian Pac J Cancer Prev 15: 2809-2813, 2014

17. Azad AK, Qiu X, Boyd K, Kuang Q, Emami M, Perera N, Palepu P, Patel D, Chen Z, Cheng D, et al: A genetic sequence variant (GSV) at susceptibility loci of 5p15.33 (TERT-CLPTM1L) is associated with survival outcome in locally advanced and metastatic non-small-cell lung cancer (NSCLC). Lung Cancer 84: 289-294, 2014.

18. Wu H and Zhu R: Quantitative assessment of common genetic variants on chromosome $5 \mathrm{p} 15$ and lung cancer risk. Tumour Biol 35: 6055-6063, 2014.

19. Zhang C, Doherty JA, Burgess S, Hung RJ, Lindström S, Kraft P, Gong J, Amos CI, Sellers TA, Monteiro AN, et al; GECCO and GAME-ON Network: CORECT, DRIVE, ELLIPSE, FOCI, and TRICL: Genetic determinants of telomere length and risk of common cancers: a Mendelian randomization study. Hum Mol Genet 24: 5356-5366, 2015.

20. Liu SG, Ma L, Cen QH, Huang JS, Zhang JX and Zhang JJ: Association of genetic polymorphisms in TERT-CLPTM1L with lung cancer in a Chinese population. Genet Mol Res 14: 4469-4476, 2015.

21. Rennert G, Kremer R, Rennert HS, Wollner M, Agbarya A, Pinchev M, Lejbkowicz F, Spitz MR and Muscat JE: Lower lung cancer rates in Jewish smokers in Israel and the USA. Int J Cancer 137: 2155-2162, 2015.

22. Kocarnik JM,Park SL, Han J,Dumitrescu L, Cheng I, Wilkens LR, Schumacher FR, Kolonel L, Carlson CS, Crawford DC, et al: Pleiotropic and sex-specific effects of cancer GWAS SNPs on melanoma risk in the population architecture using genomics and epidemiology (PAGE) study. PLoS One 10: e0120491, 2015.

23. Kachuri L, Latifovic L, Liu G and Hung RJ: Systematic review of genetic variation in chromosome $5 \mathrm{p} 15.33$ and telomere length as predictive and prognostic biomarkers for lung cancer. Cancer Epidemiol Biomarkers Prev 25: 1537-1549, 2016.

24. Karami S, Han Y,Pande M, Cheng I, Rudd J, Pierce BL, Nutter EL, Schumacher FR, Kote-Jarai Z, Lindstrom S, et al; GECCO and the GAME-ON Network: CORECT, DRIVE, ELLIPSE, FOCI, and TRICL: Telomere structure and maintenance gene variants and risk of five cancer types. Int J Cancer 139: 2655-2670, 2016. 